\title{
Printed gold for electronic applications
}

\author{
P. T. Bishop, L. J. Ashfield, A. Berzins, A. Boardman, V. Buche, J. Cookson, \\ R. J. Gordon, C. Salcianu and P. A. Sutton \\ www.goldbulletin.org
}

\begin{abstract}
Molecular and nanoparticulate gold precursors for application in inkjet printing onto flexible substrates are discussed. The choice of stabilising ligands and the size of the nanoparticles influence the solution stability of the ink and their ability to form decorative or conductive functional films.
\end{abstract}

\section{Introduction}

The use of flexible substrates creates a wealth of possibilities for the manufacture of low cost, highly functional, intelligent materials. Foreseen applications of energy efficient, lightweight, flexible devices include: RFID tags in intelligent packaging to aid product protection and manage inventory; large area displays or power sources which can be integrated into textiles to create e-paper or eye-catching displays or power generating clothing. ${ }^{1}$ A recent report has valued the market for printed and thin film electronics at $\$ 1.92$ billion in 2009 and this is predicted to grow to $\$ 335$ billion by $2029 .{ }^{2}$

Metallic thin films are primarily used in electronic devices to make electrical contacts between the semiconductors and the associated wiring. Therefore, they should exhibit conductivities of at least $10^{5} \mathrm{~S} / \mathrm{cm}$, i.e. no less than $70 \%$ of the bulk conductivity of metal. ${ }^{3}$ Preferred metals are copper, silver and gold due to their excellent conductivities. ${ }^{4}$ Of these, copper is most susceptible to degradation via oxidation, followed by silver, which is also prone to diffuse into the active material causing shortcircuits. In comparison, gold is the most resistant to tarnishing and corrosion.

Gold is used extensively in the electronics industry, particularly as a protective plating material on electrical contacts owing to its chemical inertness. Despite technological advances in electronics, gold is continuing to find application in new, large-area, lower-cost products with increased functionality such as flexible photovoltaic cells and displays. This is primarily a result of its chemical resistance and proven track record in the industry.

Traditional methods of depositing thin layers of gold include chemical vapour deposition (CVD) and magnetron sputtering. These processes are 
both time consuming and expensive as they are generally run in batch mode and carried out in high vacuum chambers or under inert atmospheres. The processes are not resource-efficient as the whole surface is coated before undergoing multiple lithographic masking and etching stages to obtain the desired pattern, which then requires additional processing to recover and recycle the metal. ${ }^{5}$

Mass-printing techniques with very high throughputs such as flexographic, gravure, offset lithography and roll-to-roll (R2R) printing techniques are especially suitable for high-volume production, providing cost-effective electronic devices. ${ }^{6}$ The flexographic technique can produce prints with resolutions below $20 \mu \mathrm{m}$ at very high speeds, ${ }^{7}$ but suffers from poor definition due to ink extrusion. ${ }^{8}$ Gravure printing has been extensively studied for printing functional materials with resolutions down to $10 \mu \mathrm{m} .{ }^{9}$ Offset lithography is less popular in printed electronics as high viscosity inks are required. ${ }^{10} \mathrm{R} 2 \mathrm{R}$ can be compatible with large flexible substrates used in printed electronics, ${ }^{11}$ but requires high initial investment. ${ }^{12}$ Screen-printing is a conventional low-cost printing technique, but cannot meet the demands for high-performance integrated circuit devices due to its low resolution.

In contrast to the other printing methods described, drop-on-demand inkjet printing can be a low cost option for preparation of functional materials and devices. ${ }^{13}$ Inkjet printing is a non-contact method that has the flexibility to produce a range of individually defined structures within the same run. This allows many of the limitations of traditional printing techniques to be overcome. These attributes allow functional ink to be deposited onto textured or pre-formed irregular surfaces and materials, such as textiles, with high precision. The pattern to be printed can also be easily tailored by simply uploading the desired design to the control module, eliminating the expense of screen manufacture and downtime associated with equipment modification. This benefit means a full device can be manufactured in one run through a series of depositions with reasonable lateral resolution in the range of tens of micrometers. ${ }^{14,15}$

When developing metal-based materials for application in printable thin film and flexible semiconducting electronic devices it is necessary for the formulated ink to be compatible with both substrates and active materials used in the devices. To meet these criteria the metal complexes or nanoparticles should be soluble in suitable organic or aqueous based media to avoid damage to the semiconductor or plastic via chemical etching. The printed films should ideally be metallised at temperatures below $150^{\circ} \mathrm{C}$, as low-cost flexible plastic substrates distort undesirably between 150 and $200^{\circ} \mathrm{C} .{ }^{16}$ Alternative methods of sintering such as laser, ${ }^{17}$ ultraviolet ${ }^{18}$ or microwave ${ }^{19}$ irradiation can be used to minimise such thermal deformation.

Early gold inks consisted of gold powder dispersed in resinous materials to produce off-bright films after firing that could be lightly burnished to produce lustrous decorative gold effects. These were typically applied by screen-printing gold ink directly onto flatwares or via water-slide transfer paper. Firing temperatures range from 500 to $950^{\circ} \mathrm{C}$ depending on the substrate. Gold mercaptide molecular complexes ${ }^{20}$ and thiol-stabilised gold nanoparticles have superseded gold powder as the main types of precursor used in gold inks. ${ }^{21}$ Johnson Matthey demonstrated that such precursors can be used as inks for inkjet printing. These inks give conductive gold tracks of width $125 \mu \mathrm{m}$ and resistivity four times greater than bulk gold when printed onto glass substrate and fired at $500^{\circ} \mathrm{C}$. The resulting films were found to adhere reasonably well onto the glass substrate, but can be used in conjunction with powders of other metals to improve colour and adhesion on ceramic or glassware. More recently, thiol stabilised nanoparticle materials have been inkjet printed onto plastic substrates to give conductive films when thermally cured at temperatures as low as $120^{\circ} \mathrm{C}^{16,22}$ or when using a chemical oxidising agent to remove the thiol ligands at room temperature. ${ }^{23}$

Further to the above, Johnson Matthey have collaborated in a number of projects concerned with the development of the next generation of metalbased inks for application in thin film electronic and photonic devices. For example, gold-based inks have been developed as part of external programmes towards thin film electronics and decorative applications. Some of the key results are presented below.

\section{Nanoparticle synthesis and their use as functional thin films}

Previous workers have prepared stabilised nanoparticles using long chain alkylamines via a one-pot aqueous synthesis. ${ }^{24}$ They can also be prepared by a two-phase synthesis in the presence of a reducing agent. ${ }^{25}$ The latter route has been 


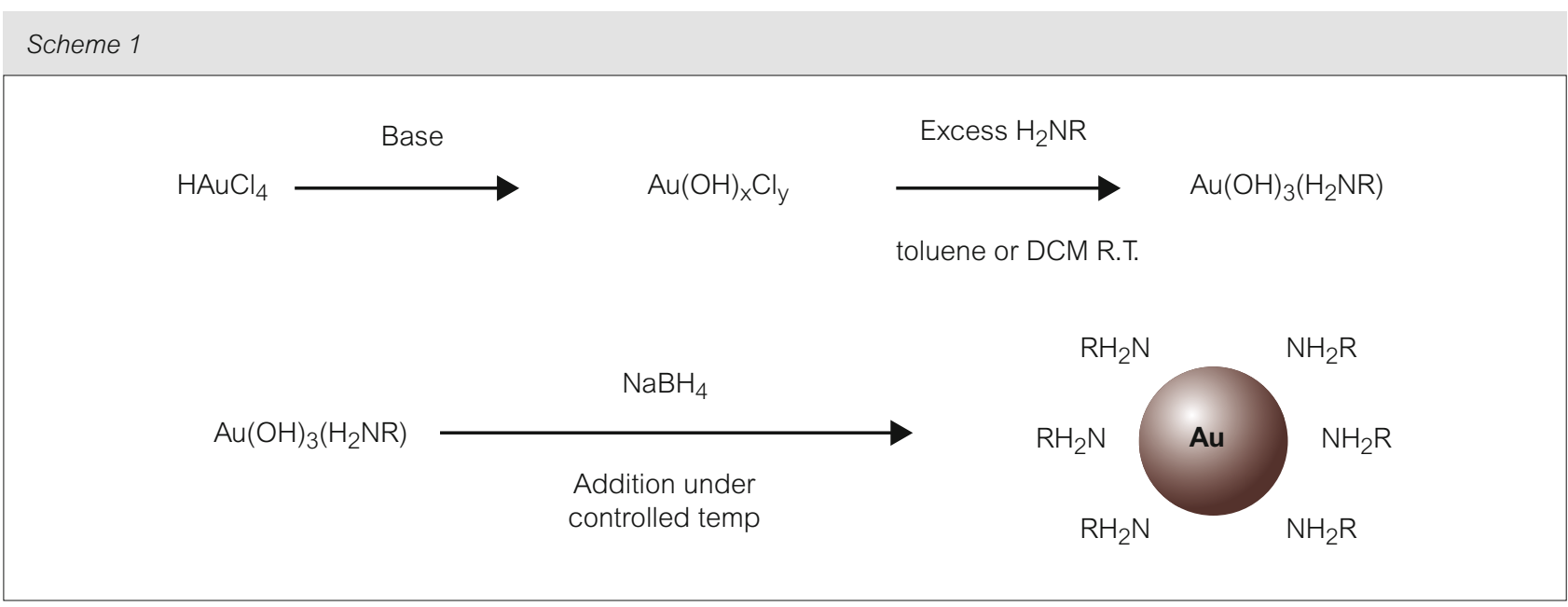

Preparation of gold-amine complex via the chloro/hydroxy precursor and its subsequent reduction using sodium borohydride to yield amine stabilised gold nanoparticles

shown to result in gold chloride ions present at the surface of the nanoparticles. ${ }^{26}$

We have discovered that halide-free aminemetal hydroxide complexes can be formed when carrying out a two-phase partitioning reaction with a gold(III) salt in the presence of a base and an organic solvent containing an amine (Scheme 1). ${ }^{27}$ The initial reaction involves controlled hydrolysis of tetrachloroaurate using sodium hydroxide to form an intermediate chloro/hydroxy species. This gold species can be extracted into a toluene solution containing excess secondary or primary amines, such as hexylamine. ${ }^{13} \mathrm{C}$ and ${ }^{1} \mathrm{H}$ NMR confirmed the presence of free and coordinated amine ligand. The amine acts as a stabilising ligand, displacing the chloro/hydroxy groups to produce a chloridefree precursor (based on elemental analysis data). This material can subsequently be used to form nanoparticles via the two-phase Brust method ${ }^{28}$ in an aqueous solution using borohydride as a reductant (Scheme 1). Particle size determination using Transmission Electron Micrograph (TEM) data indicates a size distribution of 1-6 $\mathrm{nm}$ with a mean diameter of $4 \mathrm{~nm}$.

Alternatively, a toluene solution of the intermediate Au-hexylamine complex $\left[\mathrm{Au}(\mathrm{OH})_{3}\left(\mathrm{H}_{2} \mathrm{NR}\right)\right]$ with excess hexylamine can be irradiated with UV light (125 W Hg lamp, Photochemical Reactors Ltd.) to generate nanoparticles with the same size distribution (1-6 nm) as observed for the chemical reduction route. However, the photochemical reduction resulted in a mean particle size nearer $2 \mathrm{~nm}$.
The prepared gold nanoparticles can be used to generate conductive films when spin-coated and cured thermally or under UV radiation (400 W, Hg lamp, UV-E 400B, UV Light Technology Ltd). When treated by thermal annealing, lustrous, conductive gold films are obtained at a temperature of $140^{\circ} \mathrm{C}$. The films adhere well onto plastic and remain when gently rubbed with tissue paper. They also appear to be mechanically durable to forces such as bending or flexing. A representative film is depicted in Figure 1 below.

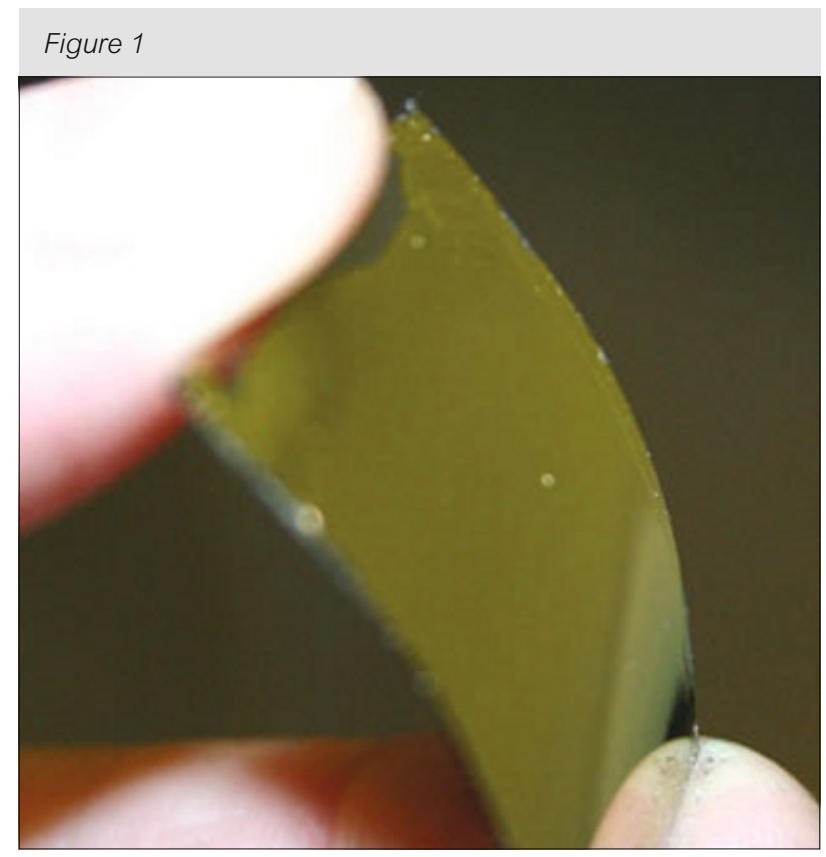

Conductive gold film obtained by spin-coating hexylamine stabilised gold nanoparticles onto a flexible plastic substrate and thermally curing at $140^{\circ} \mathrm{C}$ 
The Energy Dispersive X-ray (EDX) analysis of the film obtained via Scanning Electronic Microscopy (SEM) indicates the film is composed mainly of gold with a low level of ligand present. The sheet resistance is in the order of $10^{-2}-10^{-3} \Omega / \mathrm{sq}$, comparable to that of bulk gold. ${ }^{16}$ The high volatility of the hexylamine stabilising ligand (b.p. $\left.=132^{\circ} \mathrm{C}\right)$ is considered to be the main contributing factor to obtaining such clear conductive films, as it is easily eliminated during thermal treatment.

Decreasing the length of the alkyl group to pentylamine results in less stable nanoparticle solutions, which plate spontaneously. Whilst increasing the alkyl chain length of the amine results in more stable solutions, ligand removal is more difficult and the resistivity of the resulting films increases, ${ }^{29}$ particularly if low temperature curing is necessitated, as it is for plastic substrates. It is apparent from this work that a narrow window exists between functional conductive films and stable ink solutions. Both factors are key for industrial applications. To determine the optimal nanoparticle size required for maximum sintering and hence conductivity, the metallisation process was investigated during curing from room temperature to $500^{\circ} \mathrm{C}$ using a hot stage X-ray Diffraction (XRD).

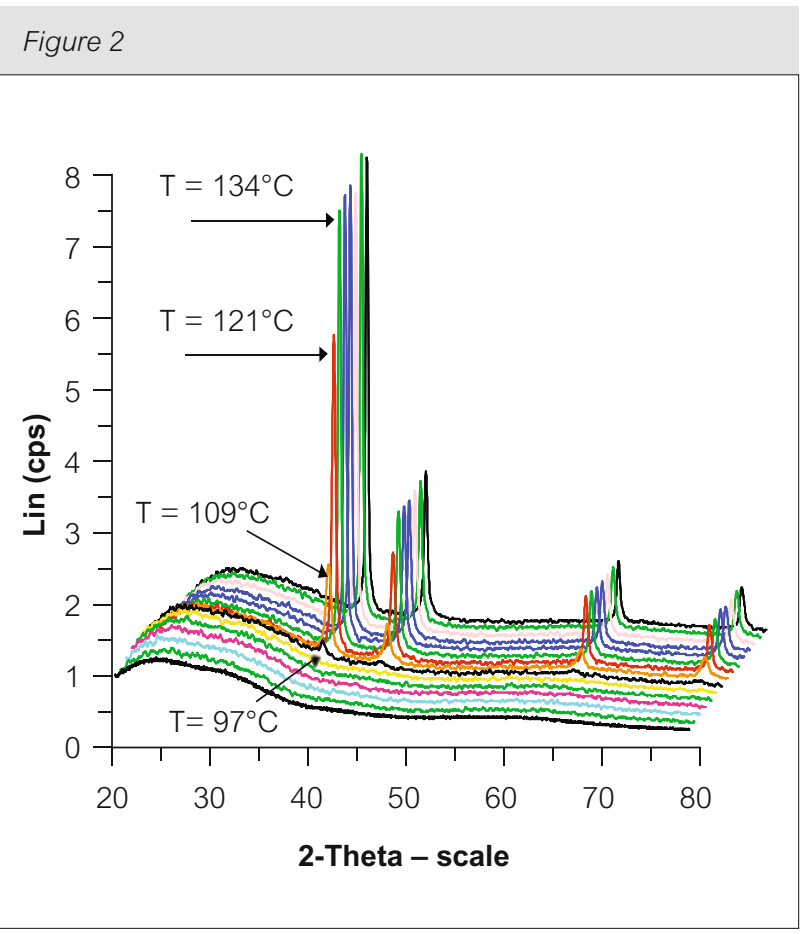

Hot-stage XRD analysis of a film spin coated onto glass of the gold-hexylamine nanoparticle solution showing metallic film formation as a function of temperature
Hot stage XRD was used to probe the crystal growth of the gold hexylamine nanoparticles during film formation. Evidence of the Au(111) phase becomes apparent in the XRD trace (Figure 2) at $97^{\circ} \mathrm{C}$, indicating the onset of gold metal formation and growth continues to $134^{\circ} \mathrm{C}$. Crystallite size grows linearly with temperature during this heating regime. The relationship between crystallite size and temperature is displayed in Figure 3, showing rapid crystallite growth up to $140^{\circ} \mathrm{C}$, the temperature at which the film becomes conductive, reaching $26 \mathrm{~nm}$ and slowing thereafter to $220^{\circ} \mathrm{C}$.

It is critical for the crystallisation process to be carefully controlled in order to fabricate films that metallise at low temperatures. Our hot-stage XRD studies show that formation of 25-30 nm crystallites produce highly conductive films. Upon increasing crystallite size, the relative surface area of the particles decreases ${ }^{30}$ thus reducing the likelihood of cold-welding occurring. Consequently, film growth could be expected to be via self-assembly and coldwelding under gentle thermal conditions rather than via further crystallite growth. This illustrates that while the presence of stabilising ligands may insulate the particles at low temperatures our results indicate that the volatility of the ligands may not be the dominant factor in the preparation of conductive films.

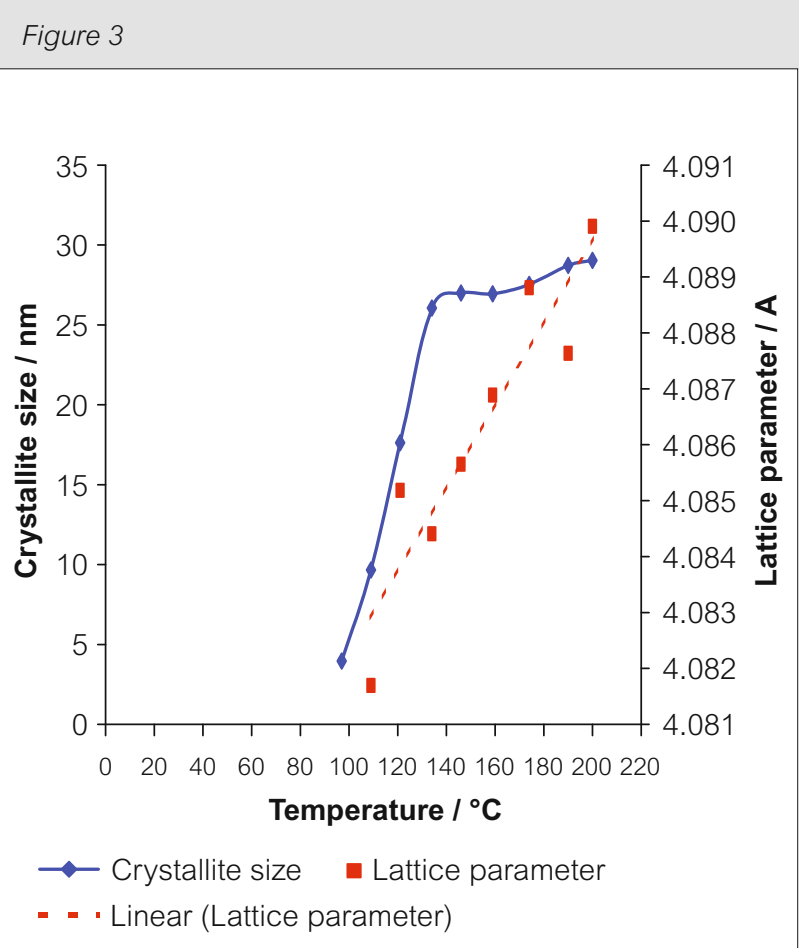

Dependence of crystallite size and lattice parameter as a function of temperature obtained by hot-stage XRD 
Scheme 2

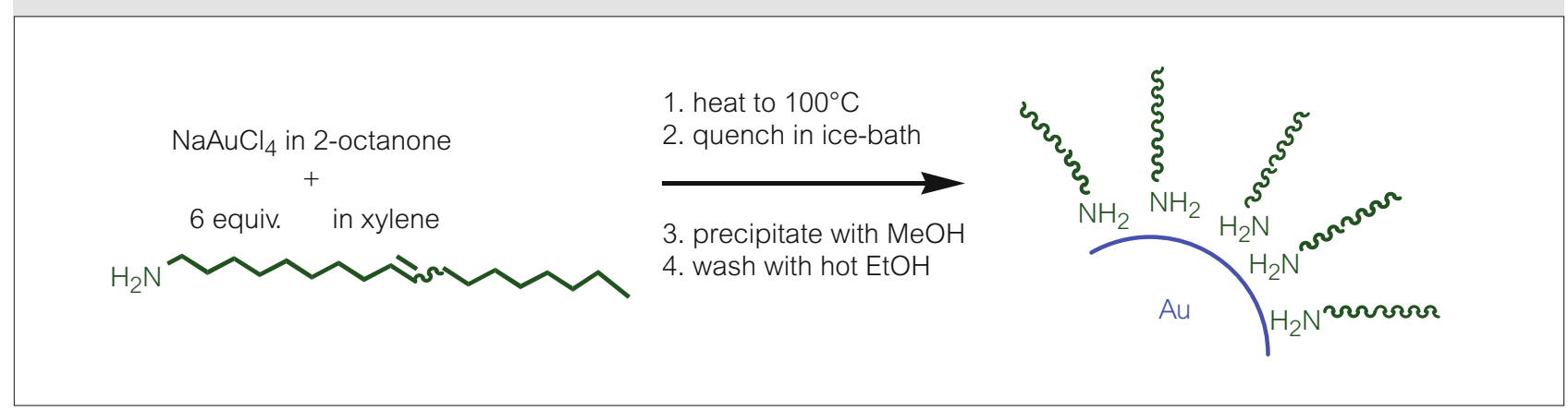

Preparation of oleylamine stabilised gold nanoparticles from sodium tetrachloroaurate

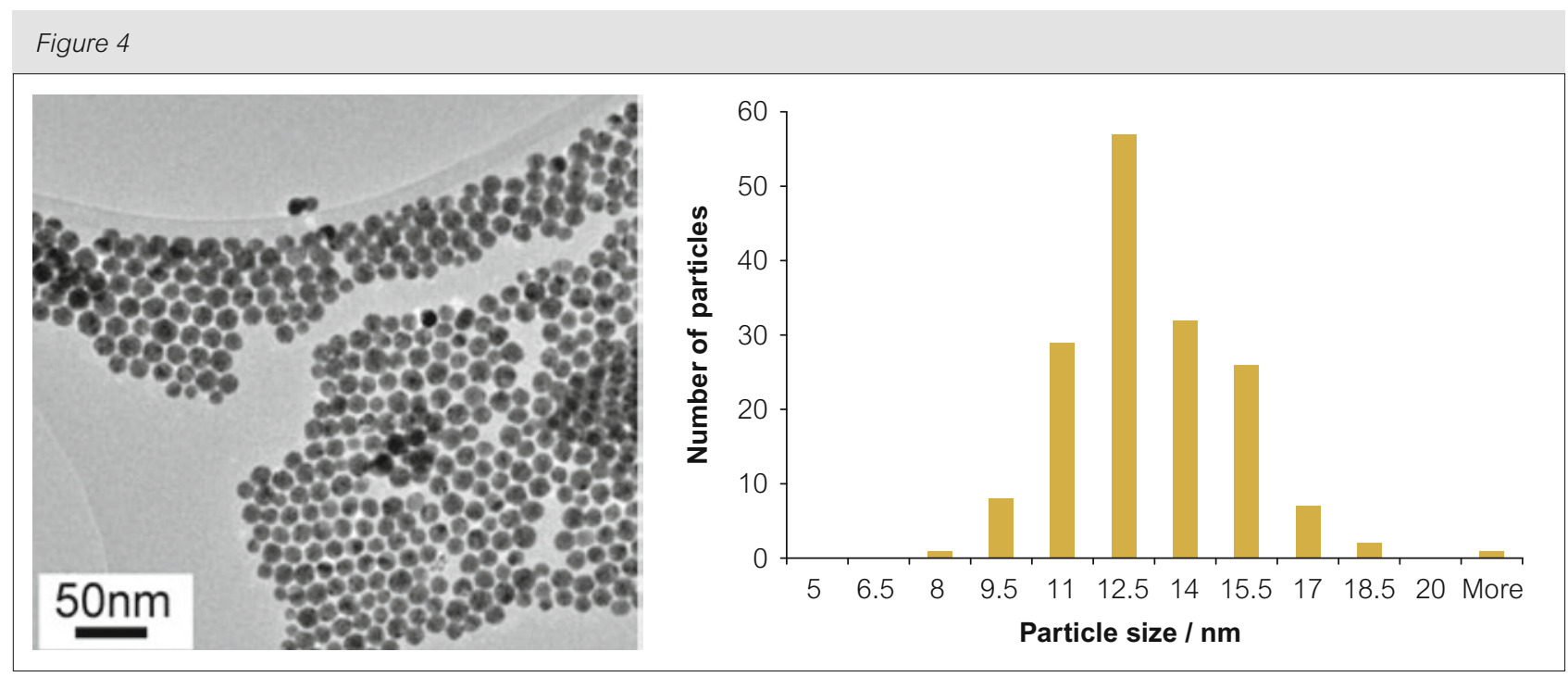

TEM micrograph and corresponding particle size distribution for the nanoparticles prepared via the route given in Scheme 2

A new series of nanoparticles were prepared according to Scheme 2. A mixture of sodium tetrachloroaurate, 2-octanone and oleylamine in xylene were heated to $100^{\circ} \mathrm{C}$. The resulting nanoparticles were precipitated using methanol. The particle size distribution and TEM data on the subsequent gold-oleylamine nanoparticles are shown in Figure 4, based on this data the average particle size is ca. $12.5 \mathrm{~nm}$. Up-scaling also gives the same particle size distributions. These particles have an Au assay of $96 \%$ (oleylamine b.p. $=350^{\circ} \mathrm{C}$ ) based on thermal analysis data.

Replacement of the gold(III) chloride precursor with $\mathrm{Au}(\mathrm{I})$-phenylacetylene in the preparation gives a chloride-free route to gold nanoparticles. ${ }^{31}$ Although the description is general, detailed studies of these reactions have allowed for scale-up to $0.5-1 \mathrm{~kg}$ batch quantities.
The bulk solids obtained from the reaction given in Scheme 2 are soluble and stable in terpenyl based solvents as well as toluene/xylene mixtures up to 30\% gold weight in solution. The solution properties and high concentration obtained were ideal to explore the inkjet properties of these materials. Initial studies involved investigating the ability to jet and produce shiny films for decorative purposes only. Printing has been achieved using a dedicated Dimatix materials printer, which is essentially a highly configurable Piezo inkjet printer. This printer demonstrated the capability to produce decorative lustrous gold print on paper at room temperature. Using suitable Piezo printing parameters to achieve consistent deposition of the gold ink, the decorative effect could be achieved in one printing pass. The printed gold surface exhibits a very directional specular reflection on smooth paper and a more decorative effect viewable at a wider angle can be achieved by printing onto specially textured paper (Figure 5 top and bottom, respectively). 


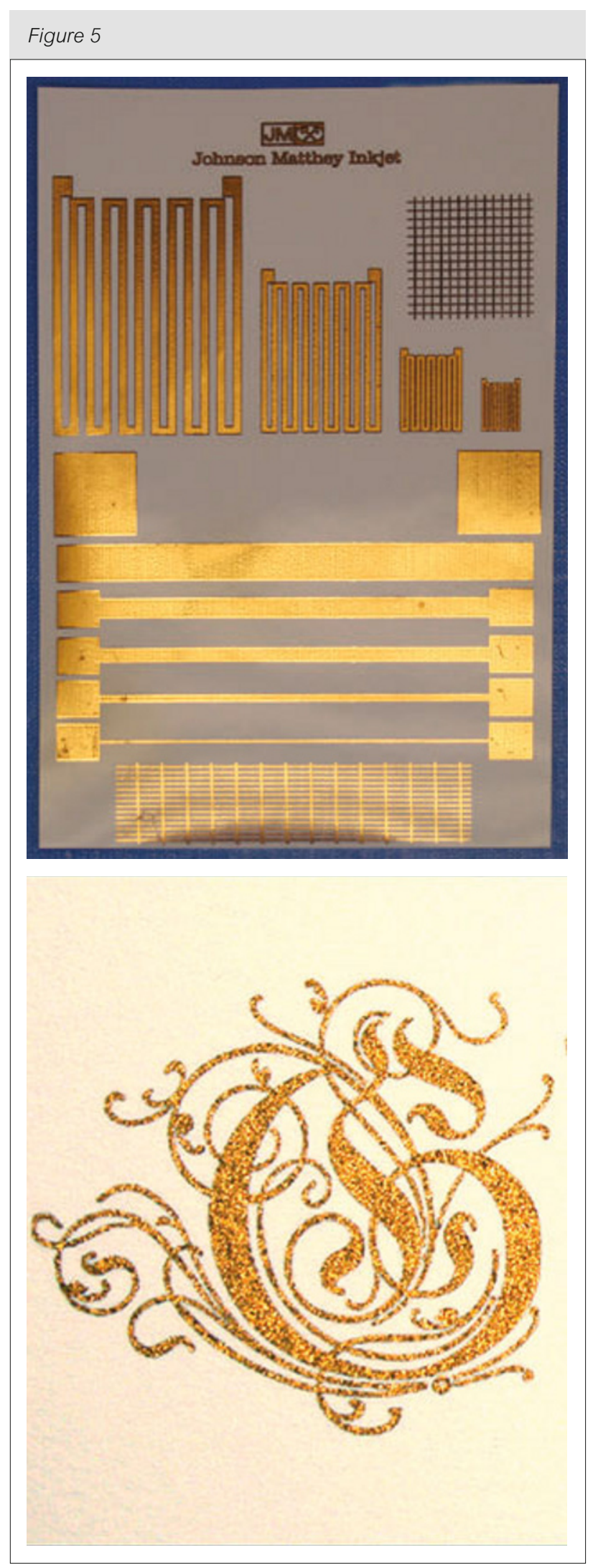

Samples of gold inkjet printed onto smooth and texture paper, top and bottom, respectively
Aside from electronic applications of gold, Johnson Matthey Technology Centre (JMTC) is continuing research into the development of gold-based inks for decorative purposes in collaboration with the World Gold Council. There is significant interest in Asia for printed gold in greeting cards and in reprints of ancient manuscripts. In collaboration with the GROW program and the Buddha Maitreya project, JMTC developed gold inks for inkjet printing onto paper. The purpose was to produce decorative pure gold text (The Prajnaparamita Sutra) on archival paper using high resolution inkjet printers. It was originally proposed to hand-write the text, however, it would have taken over 20 years to complete. Application of state-of-the-art inkjet technology was considered with a view to significantly accelerate the process. The above results go someway to addressing these needs. Although the films were decorative, the apparent coherent nature of the films made them an ideal candidate for use on plastic substrates for electronic applications.

As with the earlier work, the nanoparticles printed onto paper have to be post treated to enable properties, such as conductivity, to be developed. Heat treatment gave the best results and good sheet resistance could be established between 150 and $170^{\circ} \mathrm{C}$. Typical values for sheet resistance measured were of the order of $0.01 \Omega / s q$. Figure 6 (top) depicts a conductive circuit where OLEDs are powered via a central processing unit. These films have been prepared from the goldoleylamine nanoparticles. SEM cross-section analysis of the thermally cured film (Figure 6, bottom) indicates a high degree of densification at the surface of the paper and also the diffusion of gold nanoparticles deep into the paper. This diffusion into the polymer layers of the paper is thought to contribute to the good adhesion observed.

It appears the lower volatility of the oleylamine ligand does not adversely affect the conductive properties on porous paper substrates. However, higher process temperatures (above $200^{\circ} \mathrm{C}$ ) are required on non-porous plastic substrates to obtain conductivity comparable to that achieved on paper. Strategies are currently under investigation to lower the process temperatures. Chemical treatments of the substrate and film are areas of focus, together with controlling particle size and morphology of the gold nanoparticles. Use of non-amine containing stabilising ligands is also under study. 


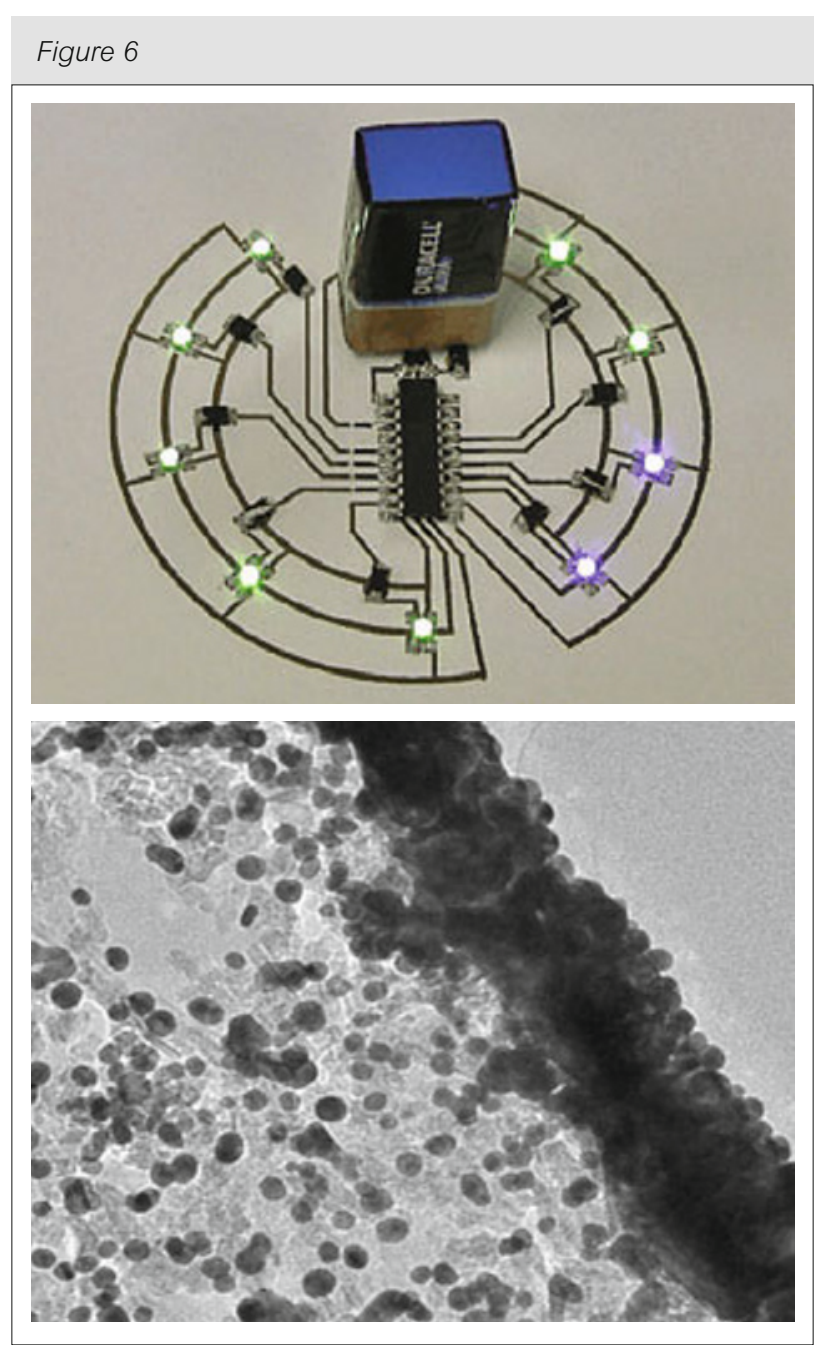

An example of a conducting circuit inkjet printed onto paper using oleylamine stabilised gold nanoparticles (top) and an SEM cross-sectional micrograph of a track on the paper (bottom)

\section{Conclusions}

This article has discussed the use of gold nanoparticles applied via inks using an inkjet printer to produce conductive films on paper substrates. Control of particle size and the stabilising ligand has provided an understanding of the optimal requirements to produce clean conductive films. Nevertheless, these inks need to be optimised for use on non-porous plastic substrates. Preliminary results have been discussed here and current work addressing these requirements is in progress.

\section{Acknowledgements}

We acknowledge the World Gold Council for financial support in the GROW program; the Technology Strategy Board (TSB) in 3D-FAB program (TP4/ $A M D / 6 / I / 22218$ ) and the EU for funding in NAIMO (FP6 project no. 500355) and ONE-P (FP7 project no. 212311).

\section{About the authors}

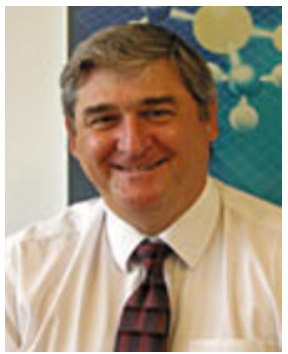

Peter Bishop, Research Manager Johnson Matthey. Peter joined Johnson Matthey in 1988 and currently holds the position of Research Manager responsible for the liquid phase catalysis and materials chemistry department at the JMTC. His group is engaged in a range of catalytic, nano-material and glass based research programs.

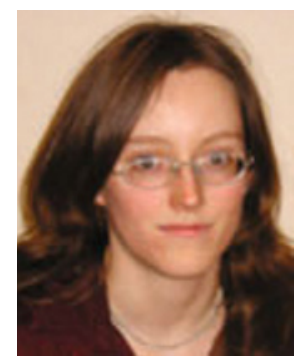

Laura Ashfield, Senior Scientist. Laura joined JM in June 2005 after completing a doctorate in inorganic chemistry at the University of Oxford. She is currently responsible for core research into the synthetic aspects of nanotechnology.

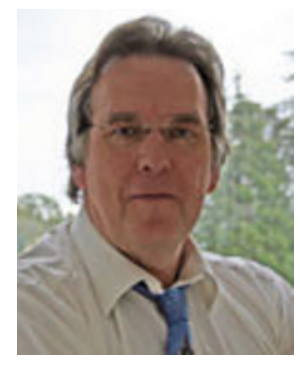

Allan Berzins, currently acting in a semi-retired role. Allan has worked for Johnson Matthey for 34 years in the UK and US. His work has involved many aspects of materials technology including thick film and semiconductor electronic applications, coatings for the aerospace industry and fuel cell development. His current interest lies in the layer fabrication and characterisation field and has involved the ink jet printing of nanomaterials.

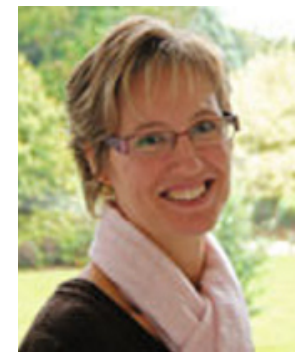

Virginie Buche, External Programmes Manager. Joined JM in 2000 and has worked on numerous projects including the area of nanotechnology. Virginie is responsible for identification of key commercial routes for new projects, opportunities and exploitation of technologies. 


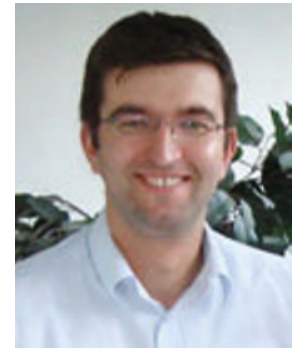

James Cookson, Senior Scientist. James joined JM in 2005 after completing a doctorate in chemistry at the University of Oxford and working at the Engineering and Physical Sciences Research Council. His main interests are in the synthesis of precious metal nanoparticles and their application in heterogeneous catalysis.

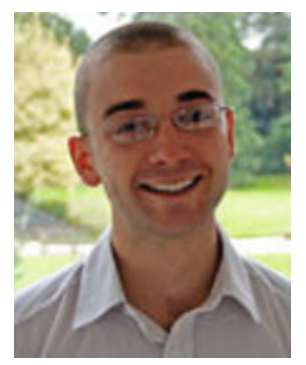

Ross Gordon, Research Scientist. Joined JM in March 2009 after completing a PhD in chemistry at the University of Edinburgh. His current research activities focus on printable metal-based nanoparticle inks for application as conductive thin films.

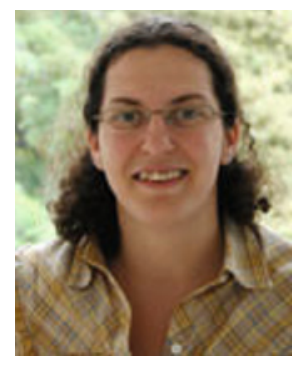

Carmen Salcianu, Research Scientist. Carmen joined JM in March 2010. She obtained her PhD degree from Cambridge University, UK. Research activities include the characterization and the applications of nanomaterials based on noble metals.

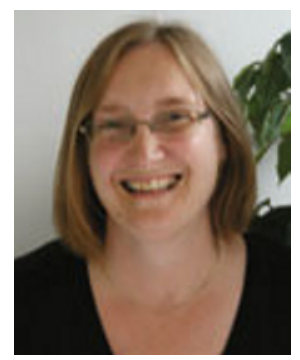

Patsy Sutton, Principal Scientist. Patsy studied gold and silver compounds as precursors for film deposition during her $\mathrm{PhD}$ and has ten years experience in gold inks for decorative applications.

\section{References}

1 Z. King, Plastic Electronics, The Advanced Institute of Management Research, 2009

2 R. Das and P. Harrop, Printed, Organic \& Flexible Electronics Forecasts, Players \& Opportunities 2009-2029, IDTechEx, 2009
3 Y. Li and J. Hou, Major Classes of Conjugated Polymers and Synthetic Strategies in Introduction to Organic

Electronics and Optoelectronic Materials and Devices, CRC Press, Boca Raton, USA, 2008

4 N.N. Greenwood and A. Earnshaw, Chemistry of the Elements, 1st ed, Pergamon Press Plc, Oxford, UK, 1990

5 A. A. R. Elshabini-Riad, Thin Film Technology Handbook, McGraw-Hill Comp. Inc., USA, 1998

6 D. R. Gamota et. al., Printed Organic and Molecular Electronics, Kluwer Academic Publishers, Massachusetts, USA, 2004

7 OE-A Roadmap for Organic and Printed Electronics, 3rd ed., ed. K. Hecker et. al., Organic Electronics Association, Frankfurt, Germany, 2009

8 R. Della Torre, US005662573A

9 G. Schmidt, Proc. SPIE, 2006, 6336, 10

10 A. C. Huebler et. al., Org. Elect., 2007, 8, 480

11 K. Allen, Flexible Flat Displays, John Wiley \& Sons Ltd., Chichester, UK, 2005

12 A. Gregg et. al., "Roll-to-Roll Manufacturing of Flexible Displays" in Flexible Flat Panel Displays, ed. G. P. Crawford, John Wiley \& Sons, New York, USA, 2005

13 P. Calvert, Chem. Mater., 2001, 13, 3299

14 H. Sirringhaus et. al., Science, 2000, 290, 2123

15 H. Sirringhaus et. al., MRS Bull., 2003, 28, 802

16 D. Huang et. al., J. Electrochem. Soc., 2003, 150, G412

17 S. H. Ko et. al., Appl. Phys. Lett., 2007, 90, 141103

18 Z. Radivojevic et. al., Proc. 12th Intl. Workshop on Thermal investigations of ICs, Nice, France, 2006

19. M. Gupta et. al., Scr. Mater., 2005, 52, 479

20 C. Corti and R. Holliday, Gold: Science and Applications, CRC Press, USA, 2010

21 A.N. Papzian, Gold Bulletin, 1982, 15, 3

22 T. Bakhishev and V. Subramanian, J. Electron. Mat., 2009, 38, 2720

23 M. J. Coutts et. al., J. Phys. Chem. C, 2009, 113, 1325.

24 M. Aslam et. al., J. Mat. Chem., 2004, 14, 1795

25 D. V. Leff et. al., Langmuir, 1996, 12, 4723

26 Kumar et. al., Langmuir, 2003, 19, 6277

27 P. T. Bishop and V. Buche, WO2006/131766 A2

28 M. Brust et. al., J. Chem. Soc., Chem. Commun., 1994, 801

29 Y. Wu et. al., Chem. Mater., 2006, 18, 4627

30 J. D. Aiken and R. G. Finke, J. Mol. Catal. A: Chem., 1999, 145, 1

31 P.T. Bishop, A. Boardman and V. Buche, WO2007/110665 A2 